10 years ESJ

Special edition

\title{
Alteración en los Hábitos del Consumidor Saltillense a Consecuencia del COVID-19
}

\author{
Dra. Adriana Méndez Wong \\ Mtra. Juana Alicia Villarreal Cavazos \\ Mtra. Elizabeth Ana Aguilar Garcés \\ Universidad Autónoma de Coahuila, Mexico
}

Doi:10.19044/esj.2021.v17n4p1

Submitted: 28 October 2020

Accepted: 22 January 2021

Published: 07 February 2021
Copyright 2021 Author(s)

Under Creative Commons BY-NC-ND

4.0 OPEN ACCESS

Cite As:

Méndez Wong A., Villarreal Cavazos J.A. \& Aguilar Garcés E.A. (2021). Alteración en los Hábitos del Consumidor Saltillense a Consecuencia del COVID-19. European Scientific Journal, ESJ, 17(4), 1. https://doi.org/10.19044/esj.2021.v17n4p1

\section{Resumen}

El estudio pretende indagar sobre la modificación en el comportamiento del consumidor saltillense debido a la contingencia por la epidemia del nuevo coronavirus COVID-19. El trabajo incluyó a 354 participantes adultos residentes en la Ciudad de Saltillo, Coahuila, México. La muestra está conformada por 195 mujeres, es decir, un $55.1 \%$ y 159 hombres lo que constituye un $44.9 \%$. Se realiza un trabajo de tipo Cuantitativo, exploratorio, descriptivo y transversal no experimental. Las preguntas son categóricas y la escala de respuesta es tipo Likert del 1 al 7, validando un instrumento de 25 elementos obteniendo un Alfa de Cronbach .786. Con base en las variables analizadas se infiere que los saltillenses se han vuelto consumidores más racionales, prefiriendo alimentos caseros, cuidando sus gastos, preocupándose por el futuro y extrañando la experiencia de comprar en tiendas físicas favoreciendo las ventas por internet y el servicio a domicilio. Y concluye que existe un nuevo perfil de consumidor no solo en Saltillo, sino a nivel mundial, que procura el bienestar en su salud, modificando hábitos alimenticios y conductuales adaptándose a esta nueva normalidad.

Palabras clave: Mercadotecnia, Comportamiento del consumidor, Tendencias 


\title{
Alteration in the Habits of the Saltillo Consumer as a Result Of COVID-19
}

\author{
Dra. Adriana Méndez Wong \\ Mtra. Juana Alicia Villarreal Cavazos \\ Mtra. Elizabeth Ana Aguilar Garcés \\ Universidad Autónoma de Coahuila, Mexico
}

\begin{abstract}
The study aims to investigate the modification in the behavior of the Saltillo consumer due to the contingency caused by the epidemic of the new coronavirus COVID-19. The work included 354 adult participants residing in the City of Saltillo, Coahuila, Mexico. The sample is made up of 195 women, that is, 55.1\% and 159 men, which constitutes 44.9\%. A quantitative, exploratory, descriptive and non-experimental cross-sectional work is carried out. The questions are categorical and the response scale is Likert type from 1 to 7, validating an instrument of 25 elements obtaining a Cronbach's Alpha .786. Based on the variables analyzed, it can be inferred that the people of Saltillo have become more rational consumers, preferring homemade foods, taking care of their expenses, worrying about the future and missing the experience of buying in physical stores, favoring internet sales and home delivery. And it concludes that there is a new consumer profile not only in Saltillo, but also worldwide, which seeks the well-being in their health, modifying eating and behavioral habits, adapting to this new normal.
\end{abstract}

Keyword: Marketing, Consumer behavior, Trends

\section{Introducción}

La llegada de la pandemia del COVID-19 a nivel mundial ha cambiado el estilo de vida de los consumidores. Como mercadólogos es muy importante conocer como los clientes han respondido a la situación, con el fin de detectar áreas de oportunidad en las estrategias desarrolladas por las empresas con fin de mantenerse en el mercado comercial.

Además, el entorno económico está dominado por escenarios pesimistas, El COVID-19 ha empujado a una profunda recesión económica a nivel mundial. La situación actual es complicada, con las restricciones de distanciamiento social se provocó una gran disminución de los empleos e ingresos. El escenario para México que reporta el informe titulado "Global Economic Forecasts: Q2 2020" (Euromonitor International, 2020) es de que la situación económica se contraerá en un $-7.9 \%$ en el 2020 , estimando un crecimiento de 1.5 para el 2021. 
Daniel Solomon y Giedrius Stalenis (2020) manifiestan que la pandemia COVID-19 producirá cambios duraderos en las actitudes y el comportamiento de los consumidores a nivel global y además el gasto mundial de los consumidores disminuirá en un $4.3 \%$ en términos reales en 2020. Así mismo se observará un aumento a largo plazo del ahorro preventivo y una disminución de las tasas de consumo. Los consumidores mayores tendrán que recurrir a las compras de comestibles en línea y el COVID-19 será un catalizador para un cambio permanente a un mundo sin efectivo.

Por su parte Alison Angus (2020) manifiesta que la pandemia ha traído las siguientes tendencias de consumo global: El uso de la tecnología en el estilo de vida para la resolución de problemas; la capacidad de las marcas para cautivar a los consumidores en menos tiempo; la movilidad limitada con consumidores cautelosos; espíritu de comunidad, pero con mayor ansiedad; búsqueda del equilibrio mental, en la nueva normalidad; casas multifuncionales y socialización virtual; productos y experiencias personalizadas; consumo local; preferencia por lo limpio, que por lo verde; queremos aire limpio en todas partes.

Según un estudio realizado en el mes de Julio del 2020, por OnePoll en conjunto con Farm Rich (Farm Rich, 2020), que analiza los hábitos alimenticios de los estadounidenses durante la pandemia de COVID-19, descubre que la persona promedio está buscando la felicidad en la comida. En estos tiempos de incertidumbre y estrés, la gente busca consuelo en los alimentos. Actividades como cocinar, compartir, experimentar y revivir recetas clásicas familiares, han traído alegría a los hogares.

Por otro lado, en España el Grupo Havas Media (2020), reportaba que, en el mes de mayo, el factor salud específicamente el contagio personal o de familiares se mostraba como el mayor temor, así como la restricción de actividades y de viajes y el no poder hacer frente a los gastos básicos. Además, se detectaba un incremento destacable en el consumo de bebidas alcohólicas y postre.

Doyle Dane Bernbach DDB México (2020) llevó a cabo un estudio sobre el perfil del nuevo ciudadano que nos dejará la crisis del coronavirus con el título ¿Cómo será el nuevo mexicano? En dicho trabajo se entrevistaron a 467 personas mayores de 18 años, quienes comentaron que el nuevo mexicano está más preocupado por su salud, que comerá mejor, y que se interesará más por contar con cobertura médica. También señala que este nuevo ciudadano les comprará a marcas que muestren mayor compromiso con los problemas medioambientales, y que apoyen al desarrollo social y mejoramiento de las comunidades en las que operan. En el trabajo se percibe una polarización política, donde un $61 \%$ considera que el gobierno ha sido un factor decisivo en agravar la crisis, mientras que un 51\% piensa que la causa 
es la infraestructura de salud con la que contamos en México. Para los mexicanos lo más difícil que se ha tenido que enfrentar durante la pandemia ha sido el miedo a resultar contagiado o que alguien cercano pueda contagiarse $(69 \%)$ y para un poco más de la mitad de la población su mayor preocupación es el perder el trabajo. Más del $70 \%$ dice que la salud y familia es lo primordial y un $21 \%$ considera que a lo que menos importancia le darán los mexicanos es a viajar. Por otra parte, se observa que las mujeres piensan en la importancia del consumo en alimento y productos de higiene, mientras que a los hombres su preocupación es proveer un techo donde vivir. Salud es alimentarse bien, y será una prioridad en el futuro cercano. Hoy los mexicanos se reafirman en pedirle a las marcas que se comprometan a trabajar por el mejoramiento del medio ambiente $(43 \%)$, que dejen de pensar sólo en lo comercial y tengan un rol en la sociedad (41\%), entre otras demandas enfocadas en el desarrollo social. Es de resaltar que un $35 \%$ de los entrevistados afirma que hará las compras de supermercados más frecuentemente de manera online o pedirá servicio a domicilio. Al preguntarles: "En una palabra, ¿cómo describirías lo que sientes en estos días frente a la situación que estás viviendo por el Coronavirus?" el 38\% de los encuestados usaron la palabra "incertidumbre", un 4.3\% "ansiedad" y un 3.8\% la palabra "impotencia".

Otro estudio realizado para conocer el ADN del consumidor post pandemia, nos refiere que en México las personas están aumentando los tentempiés entre las comidas principales, ya que anhelan momentos de placer y estimulantes. Además, que la gente ha pasado 5.5\% más tiempo en la cocina preparando sus comidas. También que la mayoría de los adultos en México, un $69 \%$, pasan tiempo jugando en sus dispositivos móviles. Por otra parte, los mexicanos cuando puedan viajar están más dispuestos a buscar alojamientos en destinos que sean ecológicos. Y por último que el 56\% de los mexicanos seguirán comprando en la tiendita de la esquina (Gonzalez, 2020).

Por tanto, la cuestión a saber es: ¿cómo han cambiado los hábitos del consumidor saltillense a consecuencia de la crisis sanitaria del COVID-19?

\section{Método}

El diseño de la investigación es de carácter exploratorio, descriptivo, cuantitativo y transversal no experimental. Los participantes son 354 sujetos con un rango de 15 a 78 años de edad, en la ciudad de Saltillo, Coahuila. El trabajo de campo se llevó a cabo de abril a mayo del 2020; la aplicación se realizó totalmente al azar, no se proporcionaron incentivos por participar; las preguntas del instrumento fueron categóricas y las respuestas se indicaron en escala de Likert de 1 a 7 donde $1=$ totalmente en desacuerdo a $7=$ totalmente de acuerdo. 


\section{Muestra}

Se determinó que la muestra de estudio sería de 384 sujetos, lo anterior con base en la ecuación 1 de Berenson y Levine (1996), para poblaciones infinitas para lo cual se asignaron valores al nivel de confianza, error muestral y probabilidad de éxitos quedando en un 95\%, 5\% y 50\%. Cabe señalar que debido a la pandemia sólo se obtuvieron 354 respuestas válidas.

$$
n=\frac{z^{2} p(1-p)}{e^{2}} \quad n=\frac{1.96^{2} \times 0.5 \times(1-0.5)}{0.05^{2}}=384
$$

\section{Muestreo}

La clase de muestreo que se llevó a cabo fue el muestreo no probabilístico, en el cual el número la selección de elementos de la población se basa, en parte, en el juicio del investigador o del entrevistador de campo (Kinnear \& Taylor, 2008).

\section{Técnica}

La técnica que se utilizó fue la encuesta autoadministrada la cual requiere de la participación directa del encuestado, llenando el cuestionario o respondiendo a las preguntas él mismo (Zikmund, 1998)

\section{Validez y confiabilidad del instrumento.}

La validez refiere al grado en que el instrumento realmente mide la variable que se pretende medir (Hernández Sampieri, Fernández Collado, \& Baptista Lucio, 2010). La validez se comprobó a través de expertos. Cabe destacar que el Cuestionario está estructurado de 25 ítems y la confiabilidad se determinó a través del programa SPSS versión 23 en español, obteniendo un Alfa de Cronbach 0.786 (tabla 1), que de acuerdo con Hair señalan una alta confiabilidad de los datos (Hair, Anderson, Tatham, \& C., 2001); (Nunnaly, 1987)

\section{Tabla 1. Estadístico de fiabilidad Alfa de Cronbach}

\begin{tabular}{cc}
\hline \hline $\begin{array}{c}\text { Alfa de } \\
\text { Cronbach }\end{array}$ & N de elementos \\
\hline .786 & 25 \\
\hline \hline
\end{tabular}

Con base en las variables de segmentación establecidas en el instrumento de recolección de datos, se consideró pertinente utilizar la prueba de análisis de la varianza de un factor (ANOVA), en la cual se examinaron, de manera sistemática, las medias de la muestra para determinar si los datos provienen de la misma población objeto de estudio, y concluir de esta manera, el nivel de significancia existente entre las variables analizadas y sus respectivos factores de segmentación. Enfatizando que se cumple con las condiciones para usar 
ANOVA de un factor, pues la variable dependiente es cuantitativa (escalar) y la variable independiente es nominal (Género).

\section{Hallazgos}

El perfil demográfico de los encuestados es que sus edades oscilan entre 15 y 70 años; el $44.9 \%$ de los entrevistados son hombres y el $51.1 \%$ son mujeres; el 5\% posee estudios de posgrado, un $41 \%$ cuenta con estudios de Licenciatura, el $32 \%$ bachillerato, $12 \%$ secundaria y $10 \%$ nivel primaria; en relación con el estado civil el $69 \%$ manifiesta ser soltero, un $24 \%$ casado, un $3 \%$ divorciado y un $4 \%$ otros. Además, un $39 \%$ son estudiantes, $34 \%$ son empleados, $14 \%$ profesionistas independientes, $9 \%$ hogar y $4 \%$ desempleado.

Tabla 2. Situación media de los consumidores con la escala ${ }^{1}$. ANOVA de un factor

\begin{tabular}{|c|c|c|}
\hline Variables & Medias & Significancia \\
\hline \multicolumn{3}{|l|}{ Hábitos de consumo } \\
\hline He permanecido en casa la mayor parte del tiempo & 4.92 & .439 \\
\hline He cambiado mis hábitos de consumo de alimentos & 4.33 & .708 \\
\hline En mi casa se ha incrementado el consumo de agua, luz. & 4.95 & .110 \\
\hline Compro más productos locales que importados & 4.83 & .653 \\
\hline Hago más ejercicio físico & 4.02 & .114 \\
\hline Estoy pidiendo comida a domicilio & 3.40 & $.045^{* *}$ \\
\hline He comprado productos en línea & 3.20 & $.051 *$ \\
\hline Extraño la experiencia de comprar en tienda física & 5.18 & $.015^{* *}$ \\
\hline Me preocupa ir a una tienda física & 4.03 & $.066^{*}$ \\
\hline Compro más en las tiendas de barrio & 4.88 & .154 \\
\hline $\begin{array}{c}\text { En mi familia hemos preferido los alimentos hechos en casa } \\
\text { en lugar de comprarlos }\end{array}$ & 5.30 & $.087 *$ \\
\hline $\begin{array}{c}\text { Elaboré mis propios productos de belleza y de higiene } \\
\text { personal de origen natural }\end{array}$ & 2.88 & .432 \\
\hline $\begin{array}{c}\text { He disminuido el consumo de carne, solo he comido } \\
\text { cuando tengo dinero para comprarla }\end{array}$ & 3.97 & $.024 * *$ \\
\hline Prefiero una dieta con base en vegetales & 3.82 & .103 \\
\hline $\begin{array}{c}\text { He pedido recomendación a amigos y familiares antes de } \\
\text { decidir una compra }\end{array}$ & 3.85 & .800 \\
\hline \begin{tabular}{|c|} 
He comprado algo influenciado por las publicaciones en \\
redes sociales
\end{tabular} & 3.68 & .265 \\
\hline $\begin{array}{c}\text { Procuro reducir mi tiempo en línea en favor de } \\
\text { experiencias de la vida real }\end{array}$ & 4.17 & .852 \\
\hline $\begin{array}{l}\text { Últimamente no comparto fotos o estados en las redes } \\
\text { sociales }\end{array}$ & 4.03 & .836 \\
\hline $\begin{array}{c}\text { A veces siento estrés digital, por el tiempo que paso en } \\
\text { redes sociales }\end{array}$ & 4.05 & .271 \\
\hline $\begin{array}{c}\text { Estoy intentando desconectarme, escogiendo dejar de lado } \\
\text { mi teléfono celular }\end{array}$ & 4.03 & .279 \\
\hline \begin{tabular}{|l|} 
Internet aumenta mi nivel de estrés diario \\
\end{tabular} & 3.95 & .267 \\
\hline
\end{tabular}




\begin{tabular}{|c|c|c|}
\hline $\begin{array}{c}\text { En general, me encuentro buscando maneras de } \\
\text { simplificar mi vida }\end{array}$ & 4.68 & .969 \\
\hline Me gusta disfrutar de la vida, pero me preocupa el futuro & 5.16 & .326 \\
\hline Soy más consciente de mis gastos & 5.19 & .315 \\
\hline $\begin{array}{c}\text { En general, los ingresos familiares han disminuido en esta } \\
\text { contingencia }\end{array}$ & 4.58 & .154 \\
\hline
\end{tabular}

${ }^{1}$ En una escala de $1=$ total desacuerdo a $7=$ total acuerdo

Diferencias estadísticamente significativas: $(*): \mathrm{p}<0.1 ;(* *): \mathrm{p}<0.05 ;(* * *): \mathrm{p}<0.01$.

Fuente: Elaboración propia

En la tabla 2 se observa que las cuatro principales variables en orden de importancia son: En mi familia hemos preferido los alimentos hechos en casa en lugar de comprarlos con una media de 5.30 en una escala de 1=total desacuerdo a 7=total acuerdo como límites, seguida de la variable Soy más consciente de mis gastos con una media de 5.19, Extraño la experiencia de comprar en tienda física, con una media de 5.18 y por último Me gusta disfrutar de la vida, pero me preocupa el futuro con una media de 5.16

$\mathrm{Al}$ mismo tiempo, se aprecia que son seis las variables en las que existen diferencias estadísticamente significativas: Estoy pidiendo comida a domicilio, He comprado productos en línea, Extraño la experiencia de comprar en tienda física, Me preocupa ir a una tienda física, En mi familia hemos preferido los alimentos hechos en casa en lugar de comprarlos y He disminuido el consumo de carne, solo he comido cuando tengo dinero para comprarla,

Se lleva a cabo un análisis comparativo en porcentajes con el programa IBM SPSS, y a continuación se presentan los resultados:

Al cuestionarles si han podido cumplir con el aislamiento social, el $42 \%$ de los participantes están de acuerdo con haber permanecido en casa la mayoría del tiempo, mientras que un $21 \%$ se le ha dificultado cumplir con esta sugerencia para prevenir el contagio.

Respecto a si han cambiado sus hábitos de consumo de alimentos, un 50\% reconoce estar de acuerdo, sin embargo, un $29 \%$ dice que continua con sus mismos hábitos al momento de comer, mientras que un $21 \%$ es indeciso en su respuesta.

Por otra parte, un $61 \%$ de los entrevistados señalan que, durante la contingencia sanitaria, en sus hogares se ha incrementado el consumo de los servicios básicos como energía eléctrica y agua potable, un $17.7 \%$ es indeciso y por el contrario un $22.3 \%$ responde que no se ha percatado de cambios en el consumo de dichos servicios.

De igual importancia, un 60\% de los participantes en el estudio prefieren adquirir más alimentos de producción local, aunque un $17.2 \%$ continúa consumiendo productos de importación. 
Otro aspecto a destacar es que se observan niveles bajos o decrecientes en la actividad física, pues así lo afirma un $43.1 \%$ de la muestra, mientras que un $39.8 \%$ realiza más ejercicio físico y un $16.7 \%$ mantiene el ritmo que tenía antes de la crisis sanitaria.

También con el confinamiento los saltillenses están pidiendo servicio a domicilio en el consumo de alimentos en un $36.2 \%$, en cambio un $52.5 \%$ no acostumbra utilizar este servicio.

Las compras a través de internet se aceleran en la pandemia, con un $41 \%$ de la muestra que así lo afirma, en comparación de un $44.8 \%$ que no opta por comprar en línea.

Además, el 40\% de los encuestados asegura extrañar la experiencia de comprar en tienda física y afirma haber disminuido sus compras en establecimientos físicos desde el inicio de la contingencia sanitaria. De igual modo un $43 \%$ asevera preocuparle asistir a una tienda física, aunque se acate las medidas sanitarias dispuestas como son el uso de gel y mascarilla, así como mantener la sana distancia. En cambio, se observa una oportunidad para las tienditas de la esquina porque su mercado meta las percibe cercanas, tal como lo demuestra un $62.7 \%$ que asegura comprar más en estas tiendas de barrio.

Con el tema de seguridad de los víveres y el ingerir alimentos nutritivos con el fin de cuidar la salud física y mental, los saltillenses están prefiriendo los alimentos caseros, de tal forma que un $68 \%$ lo confirma en sus respuestas. A diferencia de un $15 \%$ que acostumbra comer fuera de casa y un $15.8 \%$ que combina comida casera y de restaurante.

Asimismo, se detecta que un $20 \%$ de los participantes cumplen con el perfil de Eco-mixers, es decir son mujeres que deciden elaborar sus propios productos de belleza y de higiene personal con ingredientes naturales. El confinamiento y los tutoriales difundidos en las redes sociales, han modificado los hábitos de higiene de las consumidoras saltillenses, principalmente de NSE $\mathrm{AB}$ medio alto y alto.

Por otra parte, en Saltillo como ciudad norteña del país es muy frecuente contar en la dieta con el consumo de carne, pero un $40 \%$ está de acuerdo con que ha disminuido el consumo de carnes rojas debido a no contar con los recursos monetarios para adquirirla. Pero, se observa que un $35.6 \%$ de los entrevistados está prefiriendo las dietas vegetarianas, con el fin de mejorar la salud y el bienestar.

Un 38\% de los encuestados expresan que acostumbran pedir recomendación a amigos y familiares antes de decidir una compra. Y principalmente se lleva a cabo mediante la telefonía celular. Y un $35.3 \%$ ha comprado productos y servicios influenciado por las publicaciones en redes sociales. 
La pandemia ha causado estrés y ansiedad en los consumidores, y un 50\% de la muestra ha procurado reducir su tiempo en redes sociales en favor de experiencias de la vida real, mientras que un $30 \%$ manifiesta que no lo ha intentado. También, un 38\% asegura que últimamente no comparte fotos en redes sociales y un $44.3 \%$ siente a veces estrés por los contenidos digitales; concluyendo que el $42 \%$ ha intentado estar desconectado de las redes sociales y un $42.9 \%$ reconoce que el internet aumenta su nivel de estrés diario.

Otro aspecto es la incertidumbre que ocasiona la epidemia, pues al $70 \%$ le gusta disfrutar de la vida, pero le preocupa el futuro.

Respecto a si los saltillenses están disminuyendo su tasa de consumo, un $70 \%$ está de acuerdo en que se ha vuelto más consciente de sus gastos y un $50.3 \%$ asegura que en general los ingresos familiares han disminuido a consecuencia de la crisis sanitaria.

\section{Discusión}

La revisión de la literatura expone que, dentro de la contingencia global que se vive a consecuencia del COVID-19, el comportamiento del consumidor se ha modificado, el abastecimiento de víveres y servicios se presenta bajo un nuevo esquema en el que los usuarios se sientan protegidos y que, además, la economía familiar permita. El presente estudio exhibe dichos cambios en el comportamiento de la población saltillense, encuentra la importancia que éstos dan a su salud, tal como lo menciona Doyle Dane Bernbach DDB México (2020) que en su trabajo explica la inquietud del nuevo mexicano quien se preocupa más por tener cobertura médica que otras cosas, y donde se analizan las prioridades del nuevo ciudadano mexicano postpandemia.

Mofijur, Fattah, Alam, Islam, Ong, Rahman, Najafi, Ahmed, Uddin, y Mahlia, (2020) exponen en su investigación, cómo, el COVID-19 ha aumentado el sufrimiento humano, ha socavado la economía mundial, ha cambiado la vida de miles de millones de personas en todo el mundo, y la afectación significativa a la salud, la economía y el ámbito social. Dicho estudio tiene como objetivo proporcionar un amplio análisis del impacto del COVID-19 en diferentes ámbitos como lo ecológico, el sector energético, la sociedad y la economía y proporcionar información sobre las medidas preventivas globales adoptadas por gobiernos, empresas y sociedad.

Cabe destacar que es importante considerar investigaciones futuras para dominar el impacto que una pandemia puede provocar en diferentes ámbitos de forma global y, considerar que la información recabada puede proporcionar estrategias adecuadas para las tendencias que surgen ante éstas situaciones, coincidiendo con Angus (2020) quien sugiere aplicar reingeniería en la tecnología para adecuar el comportamiento del consumidor en esta nueva era, donde, aun estando en cuarentena, exista la movilidad de los usuarios, 
facilitando también el movimiento de mercancías y servicios a lo largo y ancho del mundo, con la seguridad que los consumidores reclaman ante esta pandemia.

\section{Conclusiones}

Atendiendo el propósito del estudio se encontró que los consumidores perciben cambios en sus hábitos de compra después de la pandemia del coronavirus.

Ejemplo de ello es que los saltillenses se han vuelto consumidores más racionales, prefiriendo alimentos caseros, cuidando sus gastos, preocupándose por el futuro y extrañando la experiencia que daba asistir de compras a los supermercados y la entrega fuera de las tiendas, el servicio a domicilio y la implementación de los modelos drive thru se están convirtiendo en las nuevas formas de hacer llegar los productos a los consumidores finales.

Otra muestra de los cambios de conducta en el consumidor saltillense, es el disminuir tiempo en redes sociales, para evitar el incremento de costo en servicios, ya que actualmente se utilizan para trabajo y/o estudios.

El uso de redes sociales, o estar frente a dispositivos electrónicos se ha vuelto limitado, pues los niveles de estrés se han incrementado, esto, ha provocado que los saltillenses opten por actividades en familia, que los mantenga ocupados en casa, en convivencia familiar, cambiando con eso, sus hábitos de consumo, pues han eliminado de su dieta algunos alimentos, restricciones que se han dado por razones que van desde el cambio en la alimentación para disminuir los niveles de estrés, hasta la eliminación del consumo de alimentos, como la carne, a falta de recursos económicos que son destinados para solventar otros gastos dentro del hogar.

Se concluye entonces, que prevalece una modificación en el comportamiento del consumidor saltillense, pues la actitud de éstos, ante la pandemia, que sigue evolucionando, trae consigo nuevos hábitos de compra entre la población, cuya prioridad es la salud.

Es importante seguir analizando los datos que arrojan diversos estudios en torno al comportamiento del consumidor en tiempo de pandemia, es por tanto, este estudio, una aportación que sirve de antecedente a investigaciones futuras.

\section{References:}

1. Angus, A. (2020). How is COVID-19 affecting the top 10 Global Consumer Trends 2020? Estados Unidos: Euromonitor International. Recuperado el Septiembre de 2020, de https://go.euromonitor.com/webinar-ec-2020-covid-19-impact-ongct.html 
2. Berenson, M., \& Levine, D. (1996). Estadística Básica en Administración, conceptos y aplicaciones. México: Pearson.

3. Doyle Dane Bernbach DDB México. (2020). ¿Cómo será el nuevo mexicano? México: DDB México. Obtenido de https://mcusercontent.com/1178660b5a4698f3157a92eda/files/76d69 796-50cb-466d-bede-

731db4157a8c/Resultados_Estudio_DDB_Mexico_Espan_ol_Descar gable_comprimido_.pdf

4. Euromonitor International. (2020). Global Economic Forecasts: Q2 2020. Estados Unidos: Euromonitor. Recuperado el Septiembre de 2020, de https://go.euromonitor.com/white-paper-economies-2020global-economic-forecasts-Q2.html

5. Farm Rich. (22 de Septiembre de 2020). PR Newswire. Obtenido de https://www.prnewswire.com/news-releases/2020-the-year-of-thecomfort-food-comeback-301135720.html

6. Gonzalez, F. (2020). 15 datos que podrían ser irrelevantes pero que dicen mucho del consumidor post-coronavirus. Merca2.0. Obtenido de https://www.merca20.com/15-datos-que-podrian-ser-irrelevantespero-que-dicen-mucho-del-consumidor-post-coronavirus/

7. Hair, J. F., Anderson, R., Tatham, R. L., \& C., B. W. (2001). Analisis Multivariado (5a. edición). Barcelona España: Ed. Prentice Hall.

8. Havas Media Group. (5 de Mayo de 2020). havas media. Obtenido de https://havasmedia.com/es/como-y-en-que-cambiaremos-impactodel-coronavirus-en-habitos-y-medios-estudio-de-havas-media-groupespana/

9. Hernández Sampieri, R., Fernández Collado, C., \& Baptista Lucio, P. (2010). Metodología de la investigación. México: McGraw-Hill.

10. Kinnear, T., \& Taylor, J. (2008). Investigacion de mercados. Un enfoque aplicado. McGraw Hill.

11. Mofijur, M., Fattah, I. R., Alam, M. A., Islam, A., Ong, H. C., \& Rahman, S. A. (2020). Mofijur, M., Fattah, I. R., Alam, M. A., Islam, A. S., Ong, H. C., RaImpact of COVID-19 on the social, economic, environmental and energy domains: Lessons learnt from a global pandemic. Sustainable Production and consumption, 1-44.

12. Nunnaly, J. C. (1987). Teoría Psicométrica. México: Ed. Trillas.

13. Solomon, D., \& Stalenis, G. (2020). The Implications of COVID-19 for the Global Economy. Estados Unidos: Euromonitor International. Obtenido de https://go.euromonitor.com/webinar-economies-2020covid-19-and-the-global-economy.html

14. Zikmund, W. G. (1998). Investigación de mercados. México: PrenticeHall Hispanoamericana, S.A. 